\title{
Intermediate long X-ray bursts from the ultra-compact binary candidate SLX 1737-282
}

\author{
M. Falanga ${ }^{1,2}$, J. Chenevez ${ }^{3}$, A. Cumming ${ }^{4}$, E. Kuulkers ${ }^{5}$, G. Trap ${ }^{1,6}$, and A. Goldwurm ${ }^{1,6}$ \\ ${ }^{1}$ CEA Saclay, DSM/DAPNIA/Service d'Astrophysique (CNRS FRE 2591), 91191 Gif-sur-Yvette, France \\ e-mail: mfalanga@cea.fr \\ 2 AIM - Unité Mixte de Recherche CEA - CNRS - Université Paris 7, Paris, France \\ 3 National Space Institute, Technical University of Denmark, Juliane Maries Vej 30, 2100 Copenhagen, Denmark \\ 4 Physics Department, McGill University, 3600 rue University, Montreal QC, H3A 2T8, Canada \\ 5 ISOC, ESA/ESAC, Urb. Villafranca del Castillo, PO Box 50727, 28080 Madrid, Spain \\ ${ }^{6}$ Université Paris Diderot-Paris 7 et Observatoire de Paris, Laboratoire APC, Paris, France \\ Received 2 November 2007 / Accepted 13 March 2008
}

\section{ABSTRACT}

\begin{abstract}
Aims. The low persistent flux X-ray burster source SLX 1737-282 is classified as an ultra-compact binary candidate. We compare the data on SLX 1737-282 with the other similar objects and attempt to derive constraints on the physical processes responsible for the formation of intermediate long bursts.

Methods. Up to now only four bursts, all with duration between $\simeq 15-30 \mathrm{~min}$, have been recorded for SLX 1737-282. The properties of three of these intermediate long X-ray bursts observed by INTEGRAL are investigated and compared to other burster sources. The broadband spectrum of the persistent emission in the $3-100 \mathrm{keV}$ energy band is studied with the INTEGRAL data.

Results. The persistent emission is measured to be $0.5 \%$ Eddington luminosity. From the photospheric radius expansion observed during at least one burst we derive the source distance at $7.3 \mathrm{kpc}$ assuming a pure helium atmosphere. The observed intermediate long burst properties from SLX 1737-282 are consistent with helium ignition at the column depth of 5-8 $\times 10^{9} \mathrm{~g} \mathrm{~cm}^{-2}$ and a burst energy release of $\sim 10^{41} \mathrm{erg}$. The apparent recurrence time of $\simeq 86$ days between the intermediate long bursts from SLX 1737-282 suggests a regime of unstable burning of a thick, pure helium layer slowly accreted from a helium donor star.
\end{abstract}

Key words. stars: binaries: close - stars: individual: SLX 1737-282 - stars: neutron - X-rays: bursts

\section{Introduction}

Since the first complete Galactic plane scan by Uhuru in the 70's, $\mathrm{X}$-ray missions have revealed that most neutron star low-mass X-ray binary (LMXB) systems exhibit type I X-ray bursts (Liu et al. 2007). Type I bursts are thermonuclear explosions on the surface of accreting neutron stars (NS) triggered by unstable hydrogen or helium burning. They are typically characterized by a fast rise time of $\sim 1 \mathrm{~s}$, exponential-like decays with durations ranging from seconds to minutes, and recurrence times from a few hours to days (see, e.g., Lewin et al. 1993; Strohmayer \& Bildsten 2006, for reviews). Several thousand bursts have been observed to date (see, e.g., Cornelisse et al. 2003; Galloway et al. 2006; Chelovekov et al. 2006).

Only on a few occasions, for $10 \%$ of the bursters, type I $\mathrm{X}$-ray bursts have shown decay times ranging between ten and a few tens of minutes (e.g., Swank et al. 1977; Hoffman et al. 1978; Kuulkers et al. 2002; in 't Zand et al. 2002; Molkov et al. 2005; in 't Zand et al. 2005; Chenevez et al. 2006, 2007). Such intermediate long bursts have durations and energy releases $\left(\sim 10^{41} \mathrm{erg}\right)$, intermediate between usual type I X-ray bursts, and so-called superbursts lasting more than an hour (e.g., Kuulkers 2004). Fifteen superbursts have been detected from ten sources to date (Kuulkers 2004; in 't Zand et al. 2004; Remillard \& Morgan 2005; Kuulkers 2005; Keek et al. 2007, and references therein). It is thought that unstable carbon burning (Woosley \& Taam 1976; Strohmayer \& Brown 2002) in an ocean of heavy nuclei (Cumming \& Bildsten 2001), possibly combined with photo-disintegration-triggered nuclear energy release (Schatz et al. 2003), is responsible for most superbursts.

The mechanisms driving the intermediate long bursters at very low persistent luminosity have been the subject of recent investigations (Peng et al. 2007; Cooper \& Narayan 2007), suggesting that thermally-unstable hydrogen ignition results in sporadic energetic helium bursts in a mixed hydrogen and helium environment. However, intermediate long helium bursts have also been proposed to be observed at low pure helium accretion rates (e.g., in 't Zand et al. 2005, 2007; Cumming et al. 2006).

The estimated fraction of intermediate long bursts or superbursts among the whole type I burst population is only $0.3-0.4 \%$. This shows that intermediate long bursts are very scarce events. In this paper, we report the identification of three additional intermediate long bursts from SLX 1737-282. As far as we know, this makes SLX 1737-282 the only burster source, which exclusively exhibits intermediate long bursts (four bursts all longer than 15 min observed so far, see in 't Zand et al. (2002) for the first observed burst for this source, and Sguera et al. (2007a) for the last reported burst). The present analysis concentrates on the properties of the three intermediate long bursts observed with INTEGRAL. We compare the observed intermediate long bursts with different burst types.

\subsection{The source SLX 1737-282}

Skinner et al. (1987) discovered SLX 1737-282 in 1985 with the Spacelab-2 observatory as a low persistent X-ray emission source in the energy range 3-30 keV. Since then, this source 
has been observed serendipitously during monitoring programs of the Galactic center by different X-ray observatories at the flux level of $(5-10) \times 10^{-11} \mathrm{erg} \mathrm{cm}^{-2} \mathrm{~s}^{-1}$ (Skinner et al. 1987; Sakano et al. 2002; in 't Zand et al. 2002; Tomsick et al. 2007). The most accurate position of the source has been provided by Chandra at $\alpha_{\mathrm{J} 2000}=17^{\mathrm{h}} 40^{\mathrm{m}} 42^{\mathrm{s}} .83$ and $\delta_{\mathrm{J} 2000}=-28^{\circ} 18^{\prime} 08^{\prime \prime} .4$ with an estimated accuracy of $0 .{ }^{\prime} 6$ (Tomsick et al. 2007).

From its spectral analysis and flux variability the system SLX 1737-282 was more likely classified as a low-mass X-ray binary (Sakano et al. 2002). On March 17, 2000, the discovery of a $\approx 15$ min intermediate long type I X-ray burst, with the Wide Field Camera onboard the BeppoSAX observatory, led to the classification of SLX 1737-282 as a NS low-mass Xray binary system (in 't Zand et al. 2002). The source distance was estimated to be between 5 and $8 \mathrm{kpc}$ from the burst peak flux during the radius expansion of about $6 \times 10^{-8} \mathrm{erg} \mathrm{cm}^{-2} \mathrm{~s}^{-1}$ (in 't Zand et al. 2002).

The burst detected with BeppoSAX remained the only one known from this system until the launch of the INTEGRAL satellite. A large exposure time spent by INTEGRAL on observations of the Galactic center region allowed us to detect three additional intermediate long bursts from SLX 1737-282. All three bursts had a duration of around 20-30 min. Here we study these additional intermediate long bursts.

\section{Data analysis and results}

Three intermediate long bursts have been observed by INTEGRAL/JEM-X and IBIS/ISGRI cameras (Winkler et al. 2003; Lund et al. 2003; Ubertini et al. 2003; Lebrun et al. 2003), on March 9, 2004 (burst 1), April 11, 2005 (burst 2) and April 2, 2007 (burst 3), respectively. Bursts 2 and 3 were also detected with the INTEGRAL Burst Alert System (IBAS) software (Mereghetti et al. 2003), which is dedicated to the realtime discovery and localization of gamma-ray bursts, transient $\mathrm{X}$-ray sources, and bursts in the IBIS/ISGRI data stream. One of IBAS operation modes, running on the $15-40 \mathrm{keV}$ energy interval and $10 \mathrm{~s}$ timescale, is particularly well suited to detecting type I X-ray bursts. Typically, the bursts are localized with a $\sim 3^{\prime}$ uncertainty.

We performed the data reduction with the standard Offline Science Analysis (OSA) software version 7.0. When performing analysis on a list of events collected by a coded mask telescope it is convenient, to increase the signal to noise ratio for a given source, to select events that were recorded by the parts of the detector illuminated by that source through the transparent elements of the mask. For weak sources it has been found that the best results are obtained when selection includes also partiallyilluminated detector pixels with an illumination fraction higher than about 0.4-0.5. Light curves and spectra must also be corrected for off-axis efficiency reduction due to partial modulation and variation of mask hole opacity with incidence angle, using the appropriate correction factor computed for the given source (Goldwurm et al. 2003).

The off-axis corrected burst light curves are based on events selected according to the detector illumination pattern for SLX 1737-282; for ISGRI we used an illumination threshold of 0.4 for the energy range $20-60 \mathrm{keV}$, for JEM-X we used the source events in the 3-20 keV energy range. For the persistent spectral analysis, we extracted the INTEGRAL data for all pointings within $5^{\circ}$ (JEM-X) and $9^{\circ}$ (ISGRI) of the source position for a total effective exposure of about $2.3 \mathrm{Ms}$ and $4 \mathrm{Ms}$, respectively (March 2003 to October 2006 and from the April 2007 Galactic center observation).

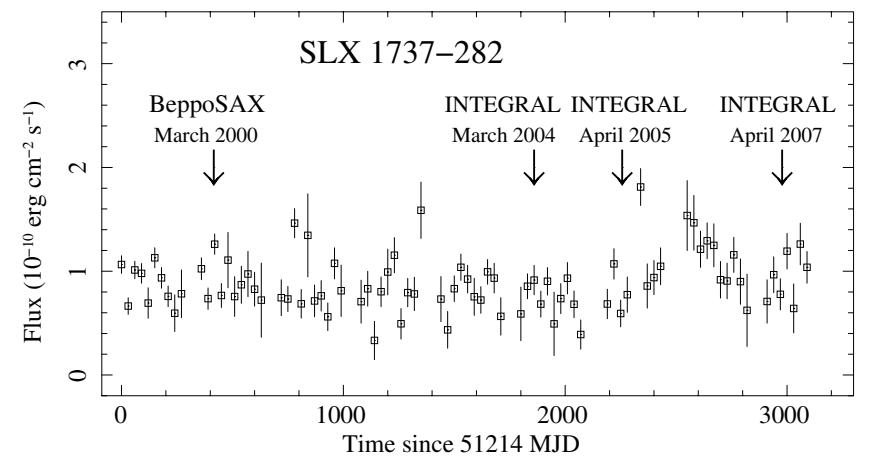

Fig. 1. RXTE bulge observations light curve for SLX 1737-282 averaged over 30-day intervals from February 5, 1999 to August 12, 2007. The $R X T E / P C A$ count rate has been converted into flux using the 2$10 \mathrm{keV}$ band Crab flux of $1.2 \times 10^{-10} \mathrm{erg} \mathrm{cm}^{-2} \mathrm{~s}^{-1}$ for $60 \mathrm{cts} \mathrm{s}^{-1}$ per 5 PCUs (in 't Zand et al. 2002). The times of the first burst, BeppoSAX observation, and the last three bursts, this work, are indicated.

Since the source is not detected in single pointings it was necessary to increase the sensitivity by combining the observations to accumulate as much exposure time as possible. Therefore, to study the weak persistent X-ray emission (see Sect. 2.1), we derived the JEM-X and ISGRI spectra from total mosaic images in four energy bands for JEM-X (3-20 keV) and five energy bands for ISGRI (20-100 keV). We applied a systematic error of $3 \%$ to combined JEM-X and ISGRI spectrum, which corresponds to the current uncertainties in the response matrices. All uncertainties in the spectral parameters are given at a $90 \%$ confidence level for single parameters.

\subsection{Persistent emission}

In Fig. 1 we plot the $2-10 \mathrm{keV}$ persistent emission for SLX 1737-282 obtained with RXTE bulge observations ${ }^{1}$ (Swank \& Markwardt 2001), which shows that the source is a weak persistent X-ray source. Since the persistent emission of SLX 1737-282 is more or less stable over the whole available data set, its average broadband spectrum has been obtained by combining the JEM-X (3-20 keV) and IBIS/ISGRI (20$100 \mathrm{keV}$ ) observations. Note that neither JEM-X nor ISGRI detected the persistent emission $10 \mathrm{ks}$ before and after the burst intervals. The energy range covered by JEM-X does not allow us to constrain the interstellar hydrogen column density, $N_{\mathrm{H}}$. We fixed in all our spectral fits $N_{\mathrm{H}}$ to $1.9 \times 10^{22} \mathrm{~cm}^{-2}$, the value found from BeppoSAX and ASCA (in 't Zand et al. 2002).

The joined JEM-X/ISGRI 3-100 keV broadband spectrum is best fitted with a photoelectrically-absorbed power-law model, with $\chi^{2} /$ d.o.f. $=8.5 / 7$, and a photon index $\Gamma=2.1 \pm 0.1$. The $3-100 \mathrm{keV}$ unabsorbed flux is $(1.3 \pm 0.15) \times 10^{-10} \mathrm{erg} \mathrm{cm}^{-2} \mathrm{~s}^{-1}$, which extrapolated in the $0.1-100 \mathrm{keV}$ band is found to be $(3.0 \pm 0.45) \times 10^{-10} \mathrm{erg} \mathrm{cm}^{-2} \mathrm{~s}^{-1}$. A multiplicative factor was included in the fit to take account of the uncertainty in the crosscalibration of the instruments. The factor was fixed at 1 for the JEM-X data and the normalizations of the ISGRI data were found within $1.2 \pm 0.1$. The best fit on the count rate spectrum and the residuals from this fit are shown in Fig. 2.

\footnotetext{
1 http://lheawww.gsfc.nasa.gov/users/craigm/galscan/ main.html
} 


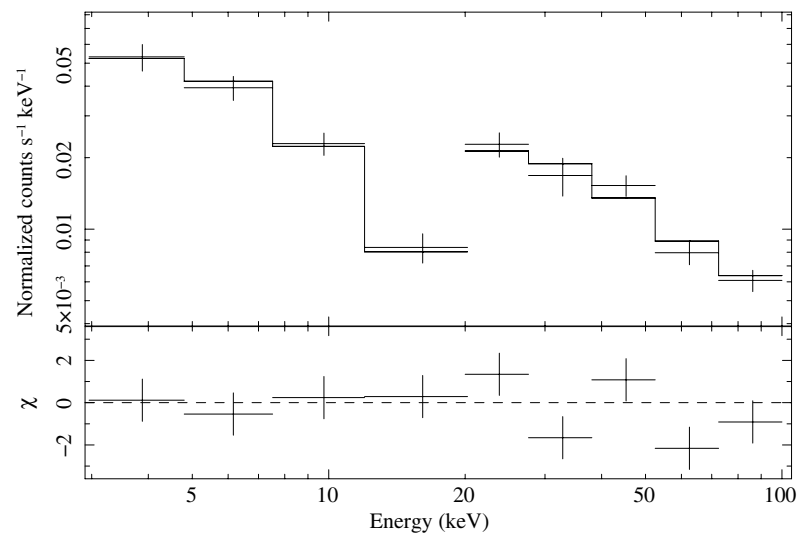

Fig. 2. INTEGRAL/JEM-X (3-20 keV) and IBIS/ISGRI (20-100 keV) averaged spectrum of SLX 1737-282 persistent emission. The best fit is obtained with a simple absorbed power-law model. The upper panel shows the data and the best fit model, whereas in the lower panel we plot the residuals from this fit.

\subsection{Burst light curves}

In Fig. 3, we show the JEM-X 3-20 keV (upper panels) and ISGRI 20-60 keV (lower panels) light curves for bursts 1, 2, and 3, respectively. Bursts 1 and 2 (see Fig. 3) were each observed over two consecutive stable pointings with a 2 min slew in between, during which no data are available. Note that burst 1 was $4.9^{\circ}$ off-axis during the first pointing, therefore for JEM-X the errors on the light curve are a factor $\sim 2$ higher compared to burst 2 and 3. Figure 4 shows that burst 2 started with a short burst-like soft event ("precursor") that lasted $\sim 6 \mathrm{~s}$, and which was more powerful in the soft energy bands. After the precursor, at higher energies, the source returned to the persistent flux level. Such behavior is typical for bursts with an extreme photospheric radius expansion and can be interpreted in terms of cooling of the NS photosphere during its expansion. After this event, the source's flux rose again to maximum, depending on the energy bands: a relatively quick increase of the intensity was first observed at low energies and then became gradually visible at higher energies. This can also be seen between JEM-X and ISGRI light curves on Fig. 3. Such behavior reflects a strong change in the hardness and is also typical for bursts with photospheric radius expansion (see, e.g., Lewin et al. 1993).

The start time for each burst was determined when the intensity rose to $10 \%$ of the peak above the persistent intensity level. The rise time is defined as the time between the start of the burst and the time at which the intensity reached $90 \%$ of the peak burst intensity. For all three bursts it was $2 \pm 1 \mathrm{~s}$; the e-folding decay time, determined over the time after the plateau, was $\tau=298 \pm 22 \mathrm{~s}, \tau=321 \pm 10 \mathrm{~s}$, and $\tau=270 \pm 14 \mathrm{~s}$, respectively for bursts 1,2 , and 3 . The total duration, i.e., from the burst start time back to the persistent flux level in the $3-20 \mathrm{keV}$ band was 25, 30, and $20 \mathrm{~min}$, respectively.

\subsection{X-ray burst spectra}

For the spectral analysis of the bursts we used JEM-X/ISGRI data in the 3-20 keV and $20-60 \mathrm{keV}$ bands, respectively. We performed time-resolved spectral analysis. The net burst spectra are well fitted by a simple BB model. The bolometric luminosity, the inferred BB temperature, $k T_{\mathrm{bb}}$, and apparent $\mathrm{BB}$ radius, $R_{\mathrm{bb}}$, at $7.3 \mathrm{kpc}$ (see Sect. 3.1) are reported in Fig. 5. In Table 1 we report the burst parameters.
The bursts fluence are obtained from the bolometric fluxes, $F_{\text {bol }}$, extrapolated in the $0.1-100 \mathrm{keV}$ energy range over the respective burst durations. The peak fluxes, $F_{\text {peak }}$, are derived from the 3-20 keV light curve peak count rates with 2 s time resolution and renormalized for the bolometric energy range.

\section{Discussion}

\subsection{Bursts, persistent flux, and source distance}

The present bursts are well described by a simple BB model representing the thermal emission from the NS surface, which is a common observed property of type I X-ray bursts (see, e.g., Galloway et al. 2006).

The light curve and spectral analysis of burst 2 show evidence for photospheric radius expansion indicating that its bolometric peak luminosity reached the Eddington limit. Assuming the Eddington luminosity for a helium burst, $L_{\text {Edd }} \approx 3.8 \times$ $10^{38} \mathrm{erg}^{-1}$, as empirically derived by Kuulkers et al. (2003), we calculate the source distance to be $7.3 \mathrm{kpc}$. For comparison, the theoretical value (e.g., Lewin et al. 1993), assuming a helium atmosphere and canonical NS parameters (1.4 solar mass and radius of $10 \mathrm{~km}$ ), leads to a source distance of $6.4 \mathrm{kpc}$. However, throughout the paper we use the $7.3 \mathrm{kpc}$ observably derived source distance. This value is in the range $5-8 \mathrm{kpc}$ inferred with the 2000 burst assuming an hydrogen or helium burst, respectively (in 't Zand et al. 2002).

The best fit to the broadband $3-100 \mathrm{keV}$ persistent emission spectrum of SLX 1737-282 required a simple power-law model with a $\Gamma \sim 2.1$. This spectral characteristic is similar to those observed in the low/hard state of LMXB (see, e.g., Barret et al. 2000; Falanga et al. 2006). Assuming a distance of $7.3 \mathrm{kpc}$ in the direction of the Galactic center for SLX 1737-282, the estimated persistent unabsorbed flux between $0.1-100 \mathrm{keV}$, $F_{\text {pers }} \approx 3 \times 10^{-10} \mathrm{erg} \mathrm{cm}^{-2} \mathrm{~s}^{-1}$, translates to a bolometric luminosity $L_{\text {pers }} \approx 1.9 \times 10^{36} \mathrm{erg} \mathrm{s}^{-1}$ or $\approx 0.5 \% L_{\text {Edd }}$. This value is consistent with the value reported by in 't Zand et al. (2002) and the fact that during the INTEGRAL observation the source flux slightly increased (see Fig. 1). This makes SLX 1737-282 another member of the class of bursters with low persistent emission (see, e.g., Cocchi et al. 2001; Cornelisse et al. 2004, and references therein). The mass accretion rate per unit area of the persistent emission, given by $L_{\text {pers }} \eta^{-1} \mathrm{c}^{-2} / A_{\text {acc }}$ (where $A_{\mathrm{acc}}=4 \pi R_{\mathrm{NS}}^{2}$ and $\eta \simeq 0.2$ is the accretion efficiency for a $1.4 M_{\odot}$ and $10 \mathrm{~km}$ radius NS), is $\dot{m}=840 \mathrm{~g} \mathrm{~cm}^{-2} \mathrm{~s}^{-1}$. Since the RXTE light curve does not indicate strong differences in the persistent flux of the source at the time of the four bursts, we are not able to comment on the exact accretion state at any time. We assume, therefore, that the bursts occurred at about the same accretion rate.

\subsection{Recurrence time of the bursts}

Up to now only four intermediate long bursts, all similar and longer than $15 \mathrm{~min}$, have been observed from the low persistent LMXB SLX 1737-282 (see Table 2). The frequency of the bursts observed with INTEGRAL, i.e., the total exposure time of $\approx 22.3 \mathrm{Ms}$ for SLX 1737-282 divided by three, (the number of observed bursts), gives 86 days. Bursts 1 and 2 have the lowest and highest total energy release: $E_{\mathrm{b}, 1} \simeq 0.7 \times 10^{41} \mathrm{erg}$ and $E_{\mathrm{b}, 2} \simeq 1.23 \times 10^{41} \mathrm{erg}$, respectively (at $7.3 \mathrm{kpc}$ distance). This corresponds to an ignition column $y=E_{\mathrm{b}}(1+z) / 4 \pi R_{\mathrm{NS}}^{2} Q_{\mathrm{nuc}}$, ranging between $y_{1,2} \approx 1.7-2.9 \times 10^{9} \mathrm{~g} \mathrm{~cm}^{-2}$ for burning 


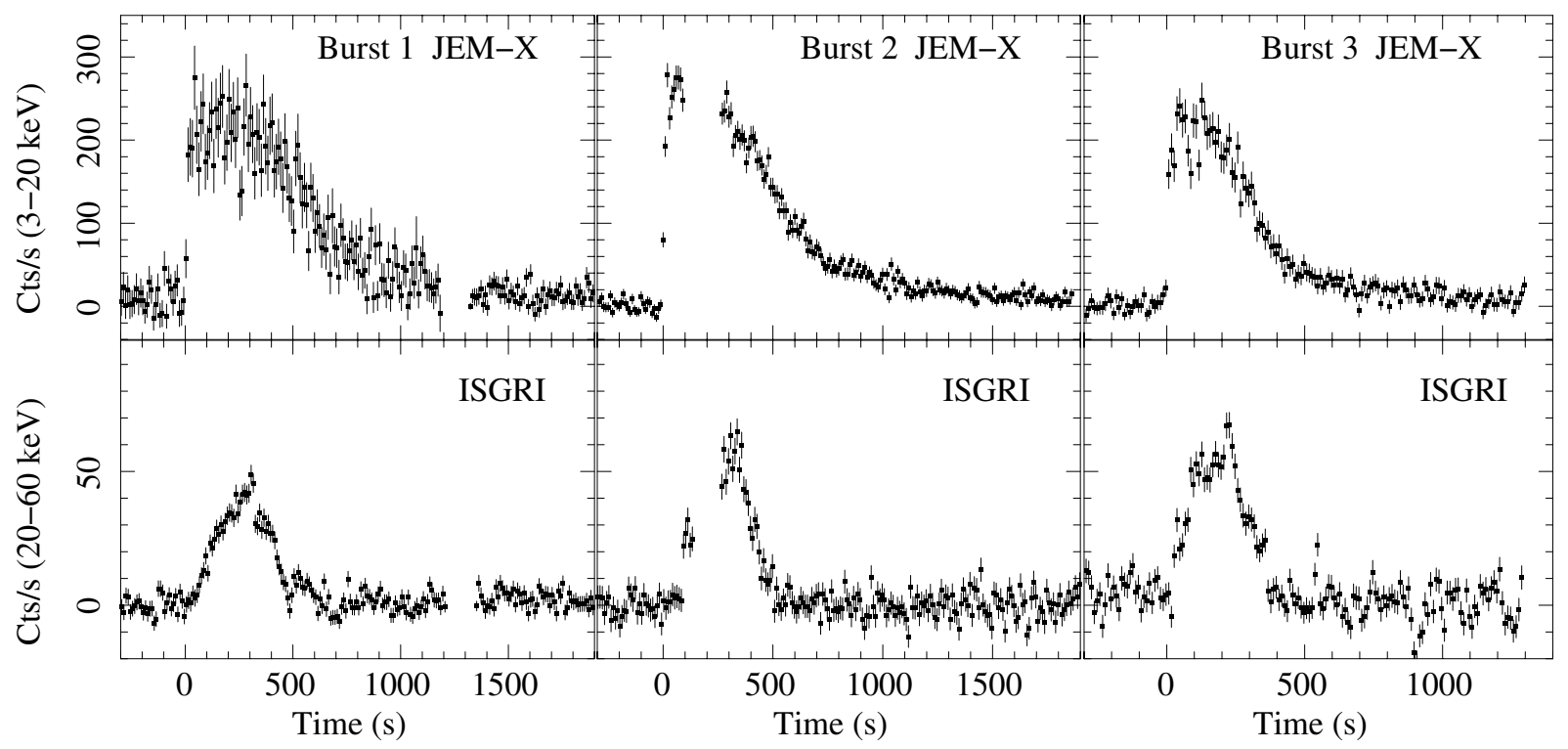

Fig. 3. The intermediate long type I X-ray bursts detected from SLX 1737-282 on March 9, 2004 (burst 1); April 11, 2005 (burst 2); and April 2, 2007 (burst 3), respectively. The time $T_{0}$ 's expressed in UTC corresponds to $17^{\mathrm{h}} 18^{\mathrm{m}} 49^{\mathrm{s}}, 08^{\mathrm{h}} 10^{\mathrm{m}} 28^{\mathrm{s}}$, and $05^{\mathrm{h}} 57^{\mathrm{m}} 8^{\mathrm{s}}$, respectively. The JEM-X $(3-20 \mathrm{keV})$ and ISGRI (20-60 keV) net light curve (background subtracted) are shown with a time bin of $10 \mathrm{~s}$. The data gap, in bursts 1 and 2, is due to a two-minute slew interval between two INTEGRAL stable pointings.

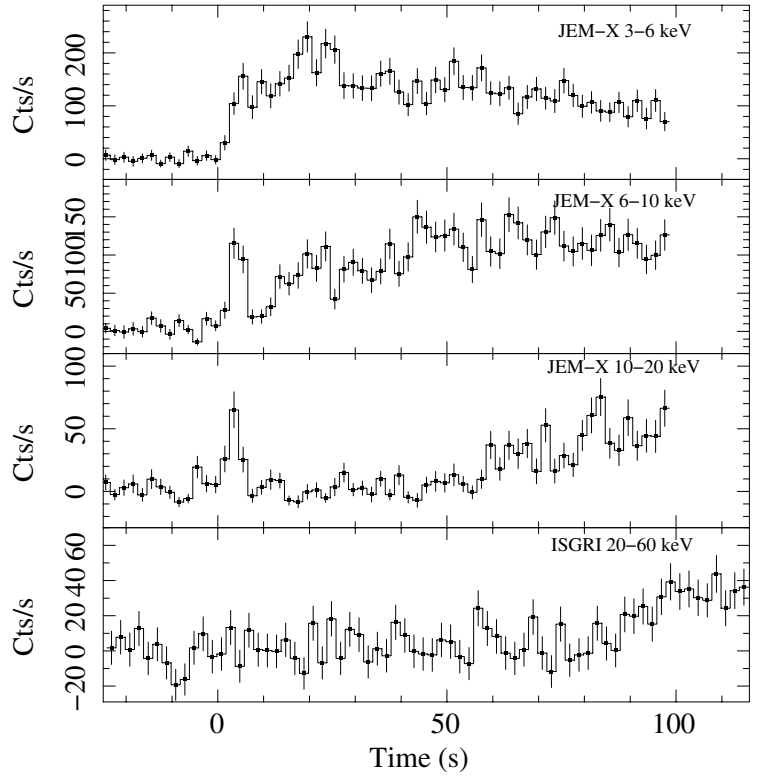

Fig. 4. Temporal profile of burst 2 (see Fig. 3) measured with JEM-X and ISGRI at different energy bands. At $T_{0}$ a soft precursor can be observed at different energy bands. The net light curve are shown with a time bin of $2 \mathrm{~s}$.

hydrogen with abundance $X=0.7$, and $y_{1,2} \approx 4.6-8.0 \times$ $10^{9} \mathrm{~g} \mathrm{~cm}^{-2}$ for $X=0$ (pure helium); here $Q_{\text {nuc }}=1.6+$ $4 X \mathrm{MeV}_{\text {nucleon }}{ }^{-1}$ is the nuclear energy release for a given average hydrogen fraction at ignition $X$, and $z=0.31$ is the appropriate gravitational redshift at the surface of a $1.4 M_{\odot}$ and $R=10 \mathrm{~km} \mathrm{NS}$. Using the relation $\Delta t_{\mathrm{rec}}=$ $y_{1,2}(1+z) / \dot{m}$ a burst recurrence time of $\approx 31-52$ days is expected for $X=0.7$, and $\Delta t_{\text {rec }} \approx 83-144$ days for pure helium burning. These values are also within the recurrence time range proposed by in 't Zand et al. (2007). The apparent recurrence time derived from our INTEGRAL observations is, therefore, more consistent with a pure helium

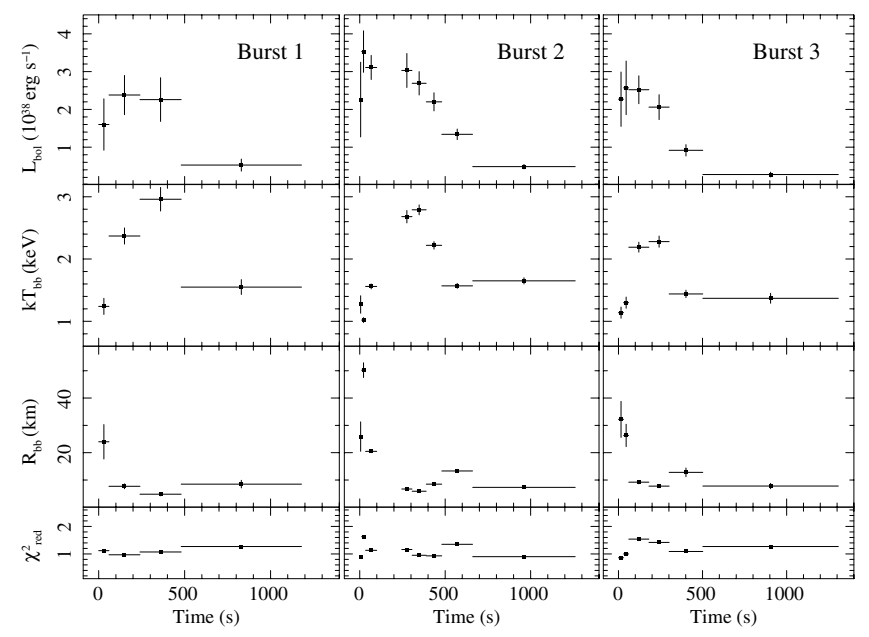

Fig. 5. The spectral evolution measured by JEM-X and ISGRI during bursts 2 and 3, respectively; bolometric luminosity at $7.3 \mathrm{kpc}$ (assuming a helium burst), radius of the photosphere and its temperature during the bursts are obtained using the BB model for the spectral fit. $\chi_{\text {red }}^{2}$ values are shown in the bottom.

burning regime. At this quite low accretion rate, hydrogen ignition would otherwise be likely (see, e.g., Boirin et al. 2007). However, observation of photospheric radius expansion is evidence for He-burning. We can also calculate the ratio of the total energy emitted in the persistent flux to that emitted in the burst $\alpha=\left(F_{\text {pers }} / f_{\mathrm{b}}\right) \Delta t_{\text {rec }}=(\gamma / \tau) \Delta t_{\text {rec }}=115-203$ for $\Delta t_{\mathrm{rec}}=86$ days and $F_{\text {pers }}=3 \times 10^{-10} \mathrm{erg} \mathrm{cm}^{-2} \mathrm{~s}^{-1}$ and $f_{\mathrm{b}}$ between $1.1-1.94 \times 10^{-5} \mathrm{erg} \mathrm{cm}^{-2}$. Assuming again that all the accreted fuel is burned during the burst, the calculated $\alpha$-value from the measurable quantities is consistent with a helium burst for $\alpha=44 M_{1.4 M_{\odot}} R_{10 \mathrm{~km}}^{-1}\left(Q_{\mathrm{nuc}} / 4.4 \mathrm{MeV} / \text { nucleon }\right)^{-1}=121$. Also in this case, a possible explanation for such an intermediate long bursting only system could be pure He burning resulting from the long accumulation of $\mathrm{He}$ at low accretion rate, possibly from a pure He donor companion (Cumming et al. 2006). 
Table 1. Burst parameters.

\begin{tabular}{llll}
\hline \hline & Burst 1 & Burst 2 & Burst 3 \\
\hline$F_{\text {peak }}^{a}\left(10^{-8} \mathrm{erg} \mathrm{cm}^{-2} \mathrm{~s}^{-1}\right)$ & $4.0 \pm 0.8$ & $6.0 \pm 0.5$ & $5.7 \pm 0.5$ \\
$f_{\mathrm{b}}^{b}\left(10^{-5} \mathrm{erg} \mathrm{cm}^{-2}\right)$ & $1.1 \pm 0.03$ & $1.94 \pm 0.02$ & $1.6 \pm 0.02$ \\
$\tau \equiv f_{\mathrm{b}} / F_{\text {peak }}(\mathrm{s})$ & $275 \pm 55$ & $323 \pm 27$ & $281 \pm 25$ \\
$\gamma \equiv F_{\text {pers }}^{c} / F_{\text {peak }}\left(10^{-3}\right)$ & $7.5 \pm 2$ & $5.0 \pm 0.8$ & $5.3 \pm 0.9$ \\
\hline
\end{tabular}

${ }^{a}$ Unabsorbed flux $(0.1-100 \mathrm{keV}) .{ }^{b}$ Fluence. ${ }^{c}$ Using the unabsorbed persistent flux $F_{\text {pers }}=(3 \pm 0.45) \times 10^{-10} \mathrm{erg} \mathrm{cm}^{-2} \mathrm{~s}^{-1}(0.1-100 \mathrm{keV})$.

The burst light curves are consistent with an ignition column depth of $7 \times 10^{9} \mathrm{~g} \mathrm{~cm}^{-2}$. Figure 6 compares the burst light curves with the cooling model of Cumming \& Macbeth (2004) in which an energy of $1.6 \times 10^{18} \mathrm{erg} \mathrm{g}^{-1}$ is deposited throughout a column of $7 \times 10^{9} \mathrm{~g} \mathrm{~cm}^{-2}$, followed by the cooling of the layer. The models are not valid for the Eddington limited part of the bursts, since they do not include the effects of radius expansion, and, therefore, allow super-Eddington luminosities. However, at late times, the models predict that the flux should decay as a power-law in time. Indeed, Fig. 6 shows that for times $\gtrsim 300$ and $\gtrsim 400$ s, following the start of burst 2 and 3, respectively, the observed decay is a power-law. The power-law fit is statistically preferred over an exponential fit by factor 1.1 and 1.5 in the $\chi_{\text {red }}^{2}$ for burst 2 and 3, respectively. The fitted power-law indices for bursts 2 and 3 are $-2.16 \pm 0.18$. This is a steeper decay than in the models, which have $F_{\text {Model }} \propto t^{-1.55}$ at late times. Further investigation is needed, but this may reflect a different dependence of the conductivity on depth, or a different initial temperature profile than assumed in the models. In Fig. 6, we have adjusted the normalization of the light curves in each case to match the observed decay. The difference in normalization factors is 2.2. Another way to explain the different decays is that the column depths of the two bursts are different, since the time at which the light curves turn over into the power-law decay depends directly on the thickness of the layer (Cumming \& Macbeth 2004). In that case, burst 3 may have an ignition depth of about a factor of two smaller than burst 2 .

\subsection{Intermediate long bursts: nuclear burning scenarios}

For a given source, the duration of the bursts depends upon the nature of the donor star and the recurrence time between the different bursts, i.e. the composition of the accreted fuel and its amount. For the high persistent sources the intermediate long bursts may be produced either by the unstable burning of a large pile of mixed hydrogen and helium, where the beta decay of the CNO cycle and rp-process is responsible for the long duration of the bursts, or from the ignition of a thick pure helium layer accumulated by the steady burning of hydrogen into helium. In the case of intermediate long bursts from low persistent bursters, they may be produced directly by the slow accretion from a pure helium donor star (Cumming et al. 2006) or if a series of weak hydrogen flashes generates a massive layer of helium that eventually ignites in an energetic pure helium flash (Peng et al. 2007; Cooper \& Narayan 2007).

The low-persistent source SLX 1737-282 is most likely an ultra-compact X-ray binary system with a helium donar star (see in 't Zand et al. 2007, and Sect. 3.5). In this picture, the intermediate long bursts from SLX 1737-282 could be associated with the pure helium flash regime accreted from a helium donor star (Cumming et al. 2006). Also, the observed burst properties from SLX 1737-282 are consistent with pure helium ignition at the column depth of $\approx 5-8 \times 10^{9} \mathrm{~g} \mathrm{~cm}^{-2}$, leading to a burst energy release of $10^{41} \mathrm{erg}$ and a recurrence time of $\approx 130$ days at $0.5 \%$ Eddington luminosity. To get ignition of helium at a depth $\approx 5-8 \times 10^{9} \mathrm{~g} \mathrm{~cm}^{-2}$ requires that the heat flux from deeper in the star (which heats the accumulating helium layer) is equivalent to approximately $1 \mathrm{MeV} /$ nucleon at $1 \%$ Eddington accretion rate (Cumming et al. 2006). Figures 18 and 19 in Cumming et al. (2006) show that this is exactly what we expect for models with slow modified URCA-like cooling in the NS core. Including our recurrence time of SLX 1737-282 on those figures would suggest a hot rather than a cold neutron star core.

The composition of the accreted material in intermediate long burst sources also showing short bursts may not necessarily be pure helium (in 't Zand et al. 2007; Chenevez et al. 2007). The presence of some hydrogen may indeed explain the differences that distinguish the short from intermediate long bursts. If a burster source exhibits both intermediate long bursts and short bursts, then a fine tuning of the accretion rate between the two regimes could be at work near the value where the accumulating hydrogen transitions from unstable burning at low accretion rates to stable burning (via the Hot $\mathrm{CNO}$ cycle) at higher accretion rates (e.g., Strohmayer \& Bildsten 2006). When stable, the hydrogen burning steadily accumulates a thick helium shell that eventually ignites. Pure helium bursts of such thick columns lead to intermediate long burst durations (e.g., Cumming et al. 2006; Peng et al. 2007). Moreover, Cumming \& Bildsten (2000) showed that for pure helium ignition, the ignition column is very sensitive to the accretion rate. In particular, the transition to unstable hydrogen burning can be quite sudden, leading to short mixed H/He bursts. As shown by Cooper \& Narayan (2007) both energetic pure helium flashes and weak hydrogen flashes may occur near the transition. These weak hydrogen bursts (undetectable because their peak luminosity is lower than the persistent luminosity) contribute to the building of the deep layer of nearly pure helium. Such weak bursts may also they trigger the ignition of the helium if its mass is sufficiently large. So, the intermediate long bursts result from the ignition of a large helium pile beneath a steady hydrogen burning shell.

\subsection{Comparison to other burster sources}

The physics of the time dependent type I X-ray bursts is associated with the thermal unstable thermonuclear reactions (e.g., Strohmayer \& Bildsten 2006). Depending on different local accretion rates, the amount of fuel, and the type of nuclear burning, we can distinguish three main burst branches, normal bursts, intermediate long bursts, and superbursts (see Fig. 7). We defined the different bursts type in Fig. 7 as follows: normal bursts are distributed along a power-law fit with index $\Gamma=-0.24$; the intermediate long bursts with a duration of $\tau=250 \mathrm{~s}$ and the superbursts with $\tau=2.8 \mathrm{~h}$. Most of the short bursts are observed at accretion rates between $0.005-0.2 \dot{M}_{\text {Edd }}$ or around the Eddington limit. For the low accretion rates $\left(\dot{M}<0.01 \dot{M}_{\text {Edd }}\right)$, these bursts are mixed $\mathrm{H} / \mathrm{He}$ burning triggered by thermally unstable $\mathrm{H}$ ignition. For intermediate accretion rates $\left(0.01 \dot{M}_{\text {Edd }} \leq \dot{M} \leq\right.$ $\left.0.02-0.07 \dot{M}_{\text {Edd }}\right)$ they are pure He shell ignition after steady $\mathrm{H}$ burning, and for high accretion rates $\left(0.02-0.07 \dot{M}_{\text {Edd }} \leq \dot{M} \approx\right.$ $\left.1 \dot{M}_{\text {Edd }}\right)$ they are $\mathrm{H} / \mathrm{He}$ burning triggered by thermally unstable He ignition (see, e.g., Strohmayer \& Bildsten 2006, and references therein). Note that in Fig. 7 we also have the relation $L_{\text {pers }} / L_{\text {Edd }} \simeq \dot{M}_{\text {acc }} / \dot{M}_{\text {Edd }}$.

All sources showing intermediate long bursts or superbursts also exhibit normal bursts, except SLX 1737-282. Sofare, the latter has only showed intermediate long bursts. In Table 2, we 
Table 2. Properties of the most powerful intermediate long bursts.

\begin{tabular}{lcccccccccc}
\hline \hline Source & \multicolumn{3}{c}{ SLX1737-282 } & SLX 1735-269 & 2S 0918-549 & IGR J17254-3257 & GX 3+1 & GX 17+2 & 4 U 1708-23 \\
\hline instrument & WFC & \multicolumn{2}{c}{ JEM-X } & JEM-X & WFC & JEM-X & JEM-X & PCA & SAS-3 \\
precursor burst & no & no & yes & no & yes & no & no & no & no & yes \\
duration (min) & 15 & 25 & 30 & 20 & 33 & 40 & 15 & 30 & $15-30$ & 25 \\
$\tau_{\text {rise }}(\mathrm{s})$ & 1 & 2 & 2 & 2 & 100 & 1 & 20 & 1.3 & $0.4-1.3$ & 20 \\
$\tau_{\text {exp }}(\min )$ & 10 & 5.0 & 5.4 & 4.5 & 10 & 3.9 & 3.7 & 10.8 & $3.2-8.3$ & 5.5 \\
$k T_{\max }(\mathrm{keV})$ & 3.0 & 3.0 & 2.8 & 2.3 & 2.9 & 3.0 & 1.6 & 2.3 & $1.8-2.4$ & 2.5 \\
$L_{\text {peak }}^{a}\left(10^{38} \mathrm{erg} \mathrm{s}^{-1}\right)$ & 3.8 & 2.5 & 3.8 & 3.6 & 5.1 & 3.5 & 0.9 & 0.8 & $1.6-2.0$ & 3.0 \\
$E_{\mathrm{b}}\left(10^{40} \mathrm{erg}\right)$ & 19 & 7 & 12 & 10 & 20 & 9 & 2.0 & 2.1 & $5.1-7.9$ & 9.7 \\
$\tau \equiv E_{\mathrm{b}} / L_{\text {peak }}(\min )$ & 8.4 & 4.7 & 5.4 & 4.7 & 6.5 & 4.3 & 3.6 & 4.4 & $5.3-6.6$ & 5.4 \\
$L_{\text {pers }}^{b}\left(\% L_{\text {Edd }}\right)$ & 0.4 & 0.5 & 0.5 & 0.5 & 1.0 & 0.6 & 0.2 & 6.0 & $75-80$ & 0.3 \\
distance $(\mathrm{kpc})$ & 8 & 7.3 & 7.3 & 7.3 & 8.5 & 5.4 & 8 & 5 & 10 & 6 \\
references & {$[1]$} & {$[2]$} & {$[2]$} & {$[2]$} & {$[3]$} & {$[4]$} & {$[5]$} & {$[6]$} & {$[7,8,9]$} & {$[10]$} \\
\hline
\end{tabular}

${ }^{a}$ Unabsorbed bolometric peak (black-body) luminosity.

${ }^{b}$ We used the bolometric unabsorbed flux from spectral fits; the observed maximum flux during radius-expansion bursts. 1. in 't Zand et al. (2002); 2. this work; 3. Molkov et al. (2005), (see also Suzuki \& Kawai 2005; Sguera et al. 2007b); 4. in 't Zand et al. (2005); 5. Chenevez et al. (2007); 6. Chenevez et al. (2006); 7. Tawara et al (1984); 8. Kuulkers et al. (2002); 9. Galloway et al. (2006); 10. Hoffman et al. (1978).

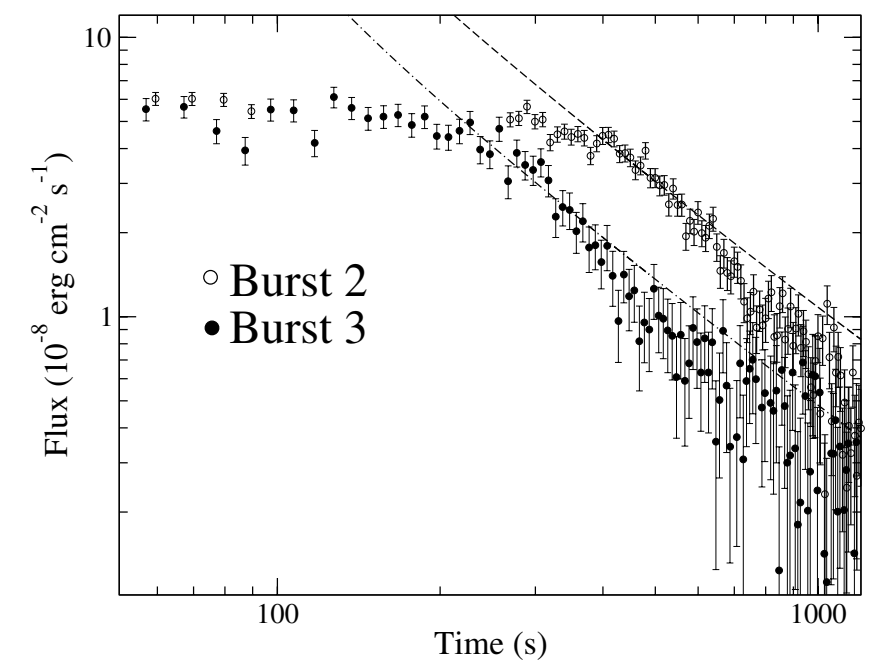

Fig. 6. Comparison of the observed decay of the bolometric black body flux (open circle burst 2 and circle burst 3 ) with a theoretical model (Cumming \& Macbeth 2004) for the cooling rate of a column of depth $7 \times 10^{9} \mathrm{~g} \mathrm{~cm}^{-2}$ and a nuclear energy release of $1.6 \times 10^{18} \mathrm{erg} \mathrm{g}^{-1}$, which is expected for helium burning to iron. The model is shown in dashed and dot-dashed curve for bursts 2 and 3 , respectively.

report the properties of the most powerful and recently studied intermediate long bursts. In Fig. 7, we also added intermediate long bursts with $\tau \approx 100 \mathrm{~s}$ from the following sources: GRS 1747-312, EXO 0748-676, and GX 17+2 (in 't Zand et al. 2003; Galloway et al. 2006). The burst properties of SLX 1737-282 are similar to the other intermediate long bursts. The intermediate long burst from SLX 1735-269 (Molkov et al. 2005 ) is the only one showing a remarkably long rise time. This was due to an extended photospheric radius expansion phase with a well separated precursor. Molkov et al. (2005) interpreted the long decay as most probably due to the mixed burning of $\mathrm{H} / \mathrm{He}$. However, in 't Zand et al. (2007) suggested this source is an ultra-compact X-ray binary system and, therefore, a pure helium burst cannot be ruled out at this relatively low accretion rate. Note that only the two high accretion rate sources, GX $3+1$ and GX $17+2$, show all three kinds of bursts, the latter being the only source that shows intermediate long bursts at the Eddington mass accretion rate (most likely resulting from mixed burning

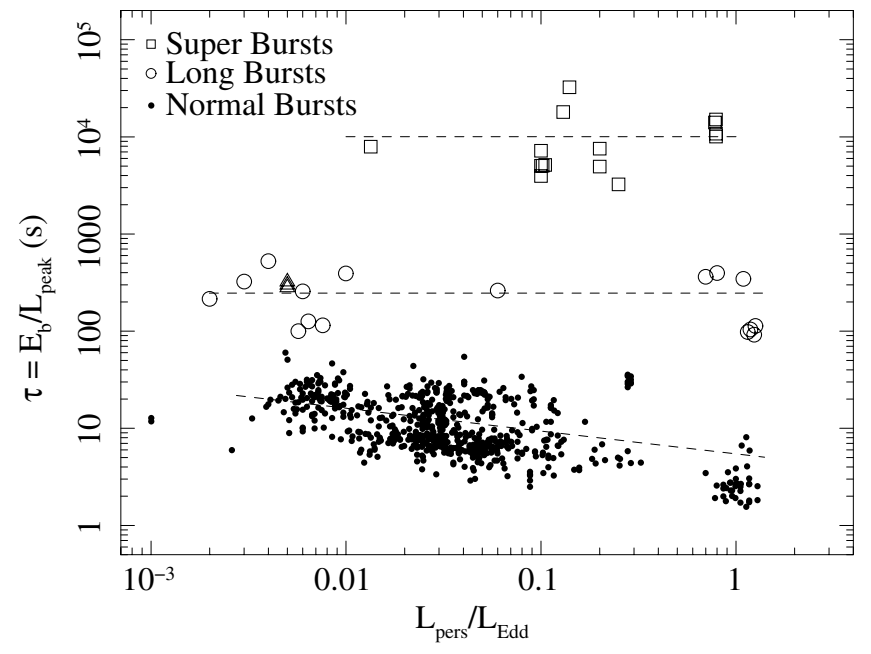

Fig. 7. Bursts effective durations vs. persistent luminosity for normal bursts (points) observed with RXTE (see Galloway et al. 2006), intermediate long bursts (open circle, and triangle for this work; see Table 2 and for the $\tau \approx 100$ s bursts see Galloway et al. 2006), and Superbursts (open square, see e.g., Kuulkers 2004; in 't Zand et al. 2004, see also Sect. 3.4).

of H/He, Chenevez et al. 2006; Galloway et al. 2006; Kuulkers et al. 2002; in 't Zand et al. 2004; Kuulkers 2002).

The superbursts are observed between $0.1-0.3 \dot{M}_{\text {Edd }}$ and the intermediate long bursts are observed between 0.002-0.01 $\dot{M}_{\text {Edd }}$, except again for GX $17+2$ at $\sim 1 \dot{M}_{\text {Edd }}$ and GX $3+1$ for the peculiar two-phase intermediate long burst at $\sim 0.06 \dot{M}_{\text {Edd }}$ (see Table 2). For Fig. 7, we derived the persistent bolometric luminosity and burst duration for the superbursts 4U 0614+09 (Kuulkers 2005) and 4U 1608-52 (Remillard \& Morgan 2005) and found $L_{\text {pers }} \approx 0.013$ and $0.14 L_{\text {Edd }}$ and $\tau=0.15 \times 10^{42} / 0.2 \times$ $10^{38} \approx 2.01 \mathrm{hr}$ and $\tau=2 \times 10^{42} / 0.6 \times 10^{38}<9.2 \mathrm{~h}$, respectively (see also for $4 \mathrm{U} 1608-52$ Keek et al. 2007). For the first time a superburst, from $4 \mathrm{U} 0614+09$, has been observed at $\sim 0.01 \dot{M}_{\mathrm{Edd}}$ mass accretion rate, which diverges from the current prediction that superbursts with carbon ignition on the hot NS crust require an accretion rate $>0.1 \dot{M}_{\text {Edd }}$ (see, e.g., Strohmayer \& Bildsten 2006). A consideration of these observations will be presented in Kuulkers (2008). However, one puzzling issue is to 


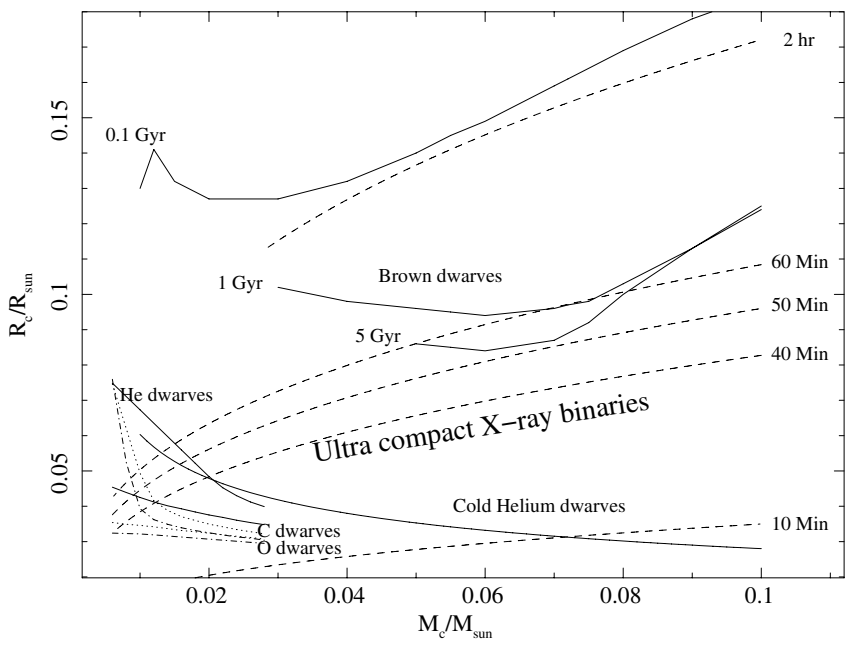

Fig. 8. Companion radius $R_{\mathrm{c}}$ vs. mass $M_{\mathrm{c}}$ plane, showing the Roche lobe constrains for the ultra-compact X-ray binaries, for $M_{\mathrm{NS}}=1.4 M_{\odot}$. The equations of state are shown for brown dwarves (solid line) and cold pure helium dwarves. The brown dwarf models are shown for different ages. The figure also shows the low-mass regime for degenerate dwarf models incorporating different compositions (dot-dash $\mathrm{O}$, dotted $\mathrm{C}$, line $\mathrm{He})$ and low $\left(10^{4} \mathrm{~K}\right)$ or high $\left(3 \times 10^{4} \mathrm{~K}\right)$ central temperatures (lower and upper curves).

understand why some sources undergo the three types of bursts and others only one or two.

\subsection{SLX 1737-282 an ultra-compact $X$-ray binary system}

The source SLX 1737-282 has recently been proposed to be an ultra-compact X-ray binary (UCXB) candidate, suggesting a pure He white dwarf donor star. An UCXB with a hydrogen poor donor star can sustain persistently low enough accretion rates, while a mixed hydrogen/helium accretor with low enough accretion rates may not exist since they then turn to be transient (in 't Zand et al. 2007).

To accrete matter persistently, the assumption of a Roche lobe-filling companion (Paczyński 1971) implies the mass-radius relation, $R_{\text {lobe }}=R_{\mathrm{c}}=1.524 \times$ $10^{-2}\left(M_{\mathrm{c}} / 1 M_{\odot}\right)^{1 / 3}\left(P_{\mathrm{orb}} / 1 \mathrm{~min}\right)^{2 / 3} R_{\odot}$, shown in Fig. 8 for different orbital periods. We divided the orbital periods into two distinct ranges - either around 10-60 -min for UCXB or $>2 \mathrm{~h}$. In the orbital period regions of 10-60 min, only very low-mass degenerate $\mathrm{O}, \mathrm{C}$, or He dwarves can be the donor star. Recent models of low-mass degenerate dwarves have been produced incorporating the effect of different compositions and temperatures (Deloye \& Bildsten 2003). The corresponding $R_{\mathrm{c}}$ versus $M_{\mathrm{c}}$ equations of state are also shown in Fig. 8. We are particularly interested in this region to study the properties of SLX 1737-282 most likely to be a pure He accretor. An additional constraint of the donor star is given by the minimum accretion rate driven by gravitational radiation in a close binary (see Verbunt \& van der Heuvel 1995, for a review). Following Bildsten \& Chakrabarty (2001) we can constrain the source companion mass as a function of the mass loss rate due to gravitational radiation, i.e., we set $0.001-0.01 \dot{M}_{\text {Edd }}=1.3163 \times$ $10^{-7}\left(M_{\mathrm{c}} / 0.01 M_{\odot}\right)^{2}\left(M_{\mathrm{NS}} / 1.4 M_{\odot}\right)^{2 / 3}\left(P_{\text {orb }} / 1 \mathrm{~min}\right)^{-8 / 3} M_{\odot} / \mathrm{yr}$ for conservative mass transfer. Note that the source distance is not a free parameter since it is determined from the Eddington luminosity reached during the burst photospheric radius expansion. Figure 8 shows that for mass accretion rates lower

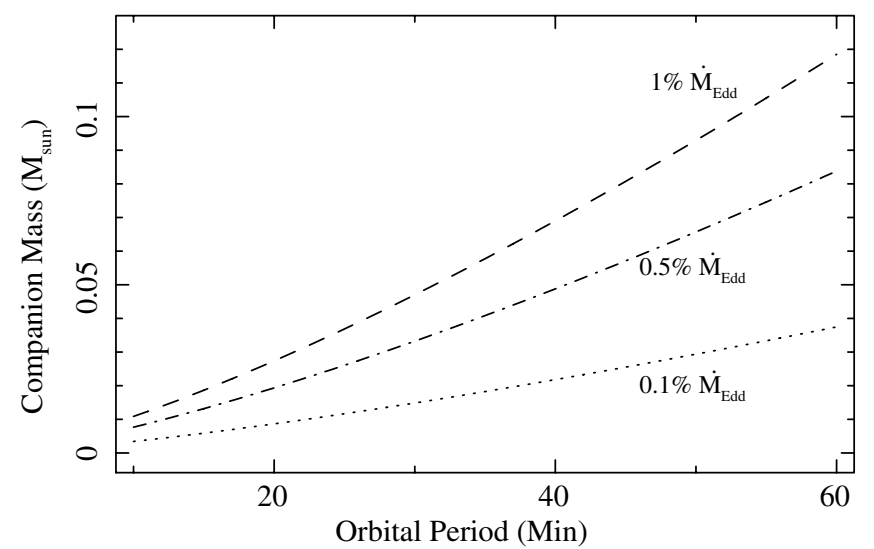

Fig. 9. Companion mass $M_{\mathrm{c}}$ vs. orbital period for different mass accretion rates, assuming that the mass transfer for UCXBs is driven by gravitational radiation, i.e., $\langle\dot{M}\rangle=\dot{M}_{\mathrm{GR}}$ The companion star mass is calculated for $1 \%, 0.5 \%$, and $0.1 \% \dot{M}_{\text {Edd }}$ mass accretion rates for orbital periods between $10-60$ min.

than $0.01 \dot{M}_{\text {Edd }}$, a pure helium donor star can be the companion star for a He only bursting source (see also in 't Zand et al. 2005, for evolutionary considerations). The knowledge of the orbital period and mass function of the system could more strongly constrain the companion star and evolution of the system, see, e.g., for the UCXB accreting X-ray millisecond pulsars like XTE J1751-305 (Markwardt et al. 2002) or XTE J1807-294 (Falanga et al. 2005). Given the mass function, $f(M)=\left(M_{\mathrm{c}} \sin i\right)^{3} /\left(M_{\mathrm{c}}+M_{\mathrm{NS}}\right)^{2}$, an important free parameter is the inclination angle of the system. Assuming an inclination angle of the binary system between the mean value of $60^{\circ}$ to a maximum value of $\sim 85^{\circ}$ the donor star has to be between 0.005 and $0.03 M_{\odot}$. This requires a low persistent mass accretion rate of $<0.01 \dot{M}_{\text {Edd }}$ as shown in Fig. 9.

In the $>2 \mathrm{~h}$ orbital period regions the donor star can be a hydrogen main-sequence star or a brown dwarf. The superburst sources belong to this region except 4U 1820-303, which has an orbital period of $\sim 11.5 \mathrm{~min}$. Most likely at variance with the UCXB intermediate long bursters, 4U 1820-303 has a very low inclination angle of between $30-35^{\circ}$ and a higher persistent emission, therefore, a more massive donor star, see Figs. 8 and 9. Also, transient accreting millisecond pulsars in this orbital period range, like SAX J1808.4-3658 or HETE J1900.1-2455 show short bursts (Galloway \& Cumming 2006; Falanga et al. 2007). For these sources, the time between bursts was long enough for hot CNO burning to significantly deplete the accreted hydrogen, so that ignition occurred in a pure helium layer underlying a stable hydrogen burning shell.

\section{Conclusions}

From an observational stand point, SLX 1737-282 is the only source that exclusively exhibits intermediate long bursts lasting about 20-30 min. These intermediate long bursts are most likely powered by the burning of a thick He layer. This is a consequence of the low accretion rate coupled most likely with the ultra-compact X-ray binary nature, which is also consistent with the lack of observed soft short bursts for SLX 1737-282. The observed properties of the helium bursts support these conclusions, also requiring $\simeq 90$ days recurrence time.

Acknowledgements. M.F. acknowledges the French Space Agency (CNES) for financial support. J.C. acknowledges financial support from ESA-PRODEX, No. 90057. 


\section{References}

Barret, D., Olive, J. F., Boirin, L., et al. 2000, ApJ, 533, 329

Bildsten, L., \& Chakrabarty, D. 2001, ApJ, 557, 292

Boirin, L., Keek, L., Méndez, M., et al. 2007, A\&A, 465, 559

Chelovekov, I. V., Grebenev, S. A., \& Sunyaev, R. A. 2006, AstL, 32, 456

Chenevez, J., Falanga, M., Brandt, S., et al. 2006, A\&A, 449, L5

Chenevez, J., Falanga, M., Kuulkers, E., et al. 2007, A\&A, 469, L27

Cocchi, M., Bazzano, A., NataLucci, L., et al. 2001, A\&A, 378, L71

Cooper, R., \& Narayan, R. 2007, ApJ, 661, 468

Cornelisse, R., in 't Zand, J. J. M., Verbunt, F., et al. 2003, A\&A, 405, 1033

Cornelisse, R., in 't Zand, J. J. M., Kuulkers, E., et al. 2004, Nucl. Phys. B (Proc. Suppl.), 132, 518

Cumming, A., \& Bildsten, L. 2000, ApJ, 544, 453

Cumming, A., \& Bildsten, L. 2001, ApJ, 559, L127

Cumming, A., \& Macbeth, J. 2004, ApJ, 603, L37

Cumming, A., Macbeth, J., in 't Zand, J. J. M., \& Page, D. 2006, ApJ, 646, 429

Deloye, C. J., \& Bildsten, L. 2003, ApJ, ApJ, 598, 1217

Falanga, M., Bonnet-Bidaud, J. M., Poutanen, J., et al. 2005, A\&A, 444, 15

Falanga, M., Götz, D., Goldoni, P., et al. 2006, A\&A, 458, 21

Falanga, M., Poutanen, J., Bonning, E. W., et al. 2007, A\&A, 464, 1069

Galloway, D. K., \& Cumming, A. 2006, ApJ, 652, 559

Galloway, D. K., Muno, M. P., Hartman, J. M., et al. 2006, ApJS, submitted [arXiv: astro-ph/0608259]

Goldwurm, A., David, P., Foschini, L., et al. 2003, A\&A, 411, L223

Hoffman, J. A., Lewin, W. H. G., Doty, J., et al. 1978, ApJ, 221, L57

in 't Zand J. J. M., Verbunt, F., Kuulkers, E., et al. 2002, A\&A, 389, L43

in 't Zand, J. J. M., Strohmayer, T. E., Markwardt, C. B., \& Swank, J. 2003, A\&A, 409, 659

in't Zand, J. J. M., Cornelisse, R., \& Cumming, A. 2004, A\&A, 426, 257

in 't Zand, J. J. M., Cumming, A., van der Sluys, M. V., Verbunt, F., \& Pols, O. R. 2005, A\&A, 441, 675

in 't Zand, J. J. M., Jonker, P. G., \& Markwardt, C. B. 2007, A\&A, 465, 953

Keek, L., in 't Zand, J. J. M., Kuulkers, E., et al. 2007, A\&A, 479, 177

Kuulkers, E. 2002, A\&A, 356, L5

Kuulkers, E. 2004, Nucl. Phys. B, 132, 466

Kuulkers, E. 2005, Astr. Tel., 483

Kuulkers, E., Homan, J., van der Klis, M., et al. 2002, A\&A, 382, 947
Kuulkers, E., den Hartog, P. R., in't Zand, J. J. M., et al. 2003, A\&A, 399, 663 Kuulkers, E., et al. 2008, in prep.

Lebrun, F., Leray, J.-P., Lavocate, Ph., et al. 2003, A\&A, 411, L141

Levine, A. M., Bradt, H., \& Cui, W. 1996, ApJ, 469, L33

Lewin, W. H. G. L., van Paradijs, J., \& Taam, R. 1993, Space Sci. Rev., 62, 223 Liu, Q. Z., van Paradijs, J., \& van den Heuvel, E. P. J. 2007, A\&A, 469, 807

Lund, N., Budtz-Jørgensen, C., Westergaard, N. J., et al. 2003, A\&A, 411, L231

Markwardt, C. B., Swank, J. H., Strohmayer, T. E., in't Zand, J. J. M., \& Marshall, F. E. 2002, ApJ, 575, 21

Mereghetti, S., Götz, D., Borkowski, R., et al. 2003, A\&A, 411, L291

Molkov, S., Revnivtsev, M., Lutovinov, A., \& Sunyaev, R. A. 2005, A\&A, 434, 1069

Paczyński, B. 1971, ARA\&A, 9, 183

Peng, F., Brown, E. F., \& Truran, J. W. 2007, ApJ, 654, 1022

Remillard, R., \& Morgan, E. 2005, Astr. Tel., 482

Sakano, M., Koyama, K., Murakami, H., Maeda, Y., \& Yamauchi, S. 2002, ApJS, 138, 19

Schatz, H., Bildsten, L., \& Cumming, A. 2003, ApJ, 583, L87

Sguera, V., Bazzano, A., Bird, A., \& Tarana, J. 2007a, Atel, 1338

Sguera, V., Bazzano, A., \& Bird, A. J. 2007b, Atel, 1340

Skinner, G. K., Willmore, A. P., Eyles, C. J., et al. 1987, Nature, 330, 544

Strohmayer, T. E., \& Brown, E. F. 2002, ApJ, 566, 1045

Strohmayer, T. E., \& Bildsten, L. 2006, in Compact stellar X-ray sources, ed. W.

H. G. Lewin, \& M. van der Klis (Cambridge: Cambridge University Press)

Suzuki, M., \& Kawai, N. 2005, Atel, 539

Swank, J., \& Markwardt, C. 2001, in New Century of X-ray Astronomy, ed. H. Inoue, \& H. Kunieda (San Francisco: ASP), ASP Conf. Ser., 251, 94

Swank, J. H., Becker, R. H., Boldt, E. A., et al. 1977, ApJ, 212, L73

Tawara, Y., Hirano, T., Kii, T., Matsuoka, M., \& Murakami, T. 1984, PASJ, 36, 861

Tomsick, J. L., Walter, R., Kaaret, P., et al. 2007, Astr. Tel., 1189

Ubertini, P., Lebrun, F., Di Cocco, G., et al. 2003, A\&A, 411, L131

Verbunt, F., \& van den Heuvel, E. P. J. 1995, in X-ray binaries, ed. W. H. G.

Lewin, J. van Paradijs, \& E. P. J. van den Heuvel (Cambridge: Cambridge University Press), 457

Winkler, C., Courvoisier, T. J.-L., Di Cocco, G., et al. 2003, A\&A, 411, L1

Woosley, S. E., \& Taam, R. E. 1976, Nature, 263, 101 